\title{
Changes on mean systemic filling pressure and microcirculation after a fluid challenge
}

\author{
HD Aya ${ }^{1,2^{*}}$, A Carsetti $^{1}$, S Pierantozzi ${ }^{1}, J$ Mellinghof ${ }^{1}$, N Fletcher $^{1,2}$, A Rhodes $^{1,2}$, M Cecconi $^{1,2}$ \\ From ESICM LIVES 2015 \\ Berlin, Germany. 3-7 October 2015
}

\section{Introduction}

In a previous study we observed that a fluid challenge of $4 \mathrm{ml} / \mathrm{Kg}$ is adequate to raise the mean systemic filling pressure (Pmsf) and to increase venous return in fluid responsive patients.

\section{Objectives}

The objective of this study is to describe the changes on microcirculation after a fluid challenge and describe the relationship with the changes on Pmsf. Our hypothesis is that changes in Pmsf could induce changes on the number of perfused vessels (PVD).

\section{Methods}

24 post-operative cardiac patients were prospectively enrolled. Arterial pressure and central venous pressure was measured invasively. Cardiac output (CO) was measured with a non-calibrated LiDCOplus monitor (LiDCO, Cambridge). Pmsf was measured using the stop-flow arterial venous equilibrium pressure method (1). Sublingual microcirculation was recorded with
CytoCam (Braedius Medical, Amsterdam, The Netherlands). Data was recorded at baseline, immediately after the fluid infusion, 5 and 10 minutes after the end of $4 \mathrm{ml} / \mathrm{Kg}$ of crystalloids infused in 5 minutes. At each time at least three sublingual microcirculation videos were acquired to assess total vessel density (TVD), microcirculatory flow index (MFI), proportion of perfused vessel (PPV), perfused vessel density (PVD) and microcirculatory heterogeneity index (MHI). Analysis was performed with AVA software v. 3.2 (MicroVision Medical, Amsterdam, The Netherlands). Responders are defined by an increase in $\mathrm{CO}$ greater than $10 \%$.

\section{Results}

Changes on haemodynamics and microcirculatory indices are presented in table 1 and 2. Pmsf increased in both groups. There were no significant changes in microcirculatory parameters, either in responders or non-responders. There was no significant correlation between changes on PVD and changes in $\mathrm{CO}(\mathrm{t}=0.08$, $p=0.59)$ or Pmsf $(\mathrm{t}=0.12, p=0.41)$.

Table 1. Changes on haemodynamics

\begin{tabular}{|c|c|c|c|c|c|c|}
\hline & \multicolumn{3}{|c|}{ Responders $(n=14)$} & \multicolumn{3}{|c|}{ Non-Responders $(n=10)$} \\
\hline & Baseline & Peak value Post-FC & $p$ & Baseline & Peak value Post-FC & $p$ \\
\hline $\mathrm{SV}(\mathrm{ml})$ & $78.5(63.3-91.3)$ & $94.5(80.3-102.8)$ & 0.001 & $69.5(57-87.5)$ & $75.5(56.5-86.8)$ & 0.1 \\
\hline$\overline{P m s f}(\mathrm{mmHg})$ & $23(16.5-26)$ & $28(20.8-29)$ & 0.01 & $25(20.8-27.5)$ & $26.5(23.8-32)$ & 0.04 \\
\hline $\mathrm{HR}(\mathrm{bpm})$ & $83(77.5-89.3)$ & $84(79.3-89.3)$ & 0.25 & $80(74.3-86.5)$ & $80(74-91)$ & 0.20 \\
\hline CVP (mmHg) & $9(7.8-11)$ & $10.5(7-12.3)$ & 0.24 & $11.5(8.5-13.5)$ & $13.5(10.3-15)$ & 0.04 \\
\hline $\mathrm{MAP}(\mathrm{mmHg})$ & $72(67.8-84.8)$ & $86.5(80.8-94)$ & 0.001 & $78(69.5-80.3)$ & $80(76.3-83.5)$ & 0.03 \\
\hline
\end{tabular}

${ }^{1}$ St George's University Hospitals NHS Foundation Trust, Adult Critical Care,

London, United Kingdom

Full list of author information is available at the end of the article 
Table 2. Changes on microcirculatory indices

\begin{tabular}{lcccccc}
\hline & \multicolumn{2}{c}{ Responders $(\mathbf{n}=\mathbf{1 4})$} & \multicolumn{2}{c}{ Non-Responders $(\mathbf{n}=\mathbf{1 0})$} \\
\hline MFI & Baseline & Peak value Post-FC & $p$ & Baseline & Peak value Post-FC & $p$ \\
\hline TVD $(\mathrm{n} / \mathrm{mm})$ & $2.83(2.66-3)$ & $2.83(2.5-3)$ & 0.75 & $2.92(2.56-3)$ & $2.85(2.35-2.95)$ & 0.21 \\
\hline PVD $(\mathrm{n} / \mathrm{mm})$ & $10.8(9-12.6)$ & $12(10.2-12.9)$ & 0.3 & $12.6(11.4-13)$ & $12.1(9.5-14.4)$ & 0.79 \\
\hline PPV $(\%)$ & $9.9(8.8-12.4)$ & $11.6(9.9-12.6)$ & 0.55 & $12(10.8-12.7)$ & $11.2(8.7-14)$ & 0.39 \\
\hline MHI & $98.3(94.8-99.2)$ & $95.6(90-6-99.1)$ & 0.47 & $95.5(94.6-97.4)$ & $92.8(89.2-98.7)$ & 0.17 \\
\hline
\end{tabular}

\section{Conclusions}

Despite the changes in Pmsf and macrohaemodynamics, a fluid challenge does not improve microcirculation in patients after cardiac surgery.

\section{Authors' details}

'St George's University Hospitals NHS Foundation Trust, Adult Critical Care, London, United Kingdom. ${ }^{2}$ St George's University of London, Cardiovascular Institute, London, United Kingdom.

Published: 1 October 2015

\section{Reference}

1. Aya HD, Rhodes A, Fletcher N, et al: Transient stop-flow arm arterialvenous equilibrium pressure measurement: determination of precision of the technique. J Clin Monit Comput 2015.

doi:10.1186/2197-425X-3-S1-A20

Cite this article as: Aya et al:: Changes on mean systemic filling pressure and microcirculation after a fluid challenge. Intensive Care Medicine Experimental 2015 3(Suppl 1):A20.

\section{Submit your manuscript to a SpringerOpen ${ }^{\circ}$ journal and benefit from:}

- Convenient online submission

- Rigorous peer review

- Immediate publication on acceptance

- Open access: articles freely available online

- High visibility within the field

- Retaining the copyright to your article

Submit your next manuscript at $>$ springeropen.com 